\title{
INTERPLAY BETWEEN AIR AND WATER
}

\author{
Leen van Wijngaarden \\ J.M.Burgers Centre for Fluid Dynamics \\ University of Twente, Enschede, The Netherlands. \\ I.vanwijngaarden@tn.utwente.nl
}

\begin{abstract}
In the Prologue I recall, among others, the period of the Cold War in which, thanks to Polish colleagues, scientific contacts between East and West were maintained. After that, several aspects of the flow of mixtures of air and water will be discussed and illustrated by examples.

Finally I will give some comments on the differences and similarities between fundamental and applied science and scientists.
\end{abstract}

Keywords: Multiphase flow, bubbly flows

\section{Prologue}

It is a great honour to be invited to deliver the Opening Lecture at ICTAM 2004, especially now that it is here in Warsaw, a city of great significance for Mechanics. It reminds me of the Cold War when East was East and West was West. They could nevertheless meet here in Poland, where Władek Fiszdon organized once in two years a "Symposium on Advanced Problems and Methods in Fluid Mechanics". Participation was on invitation and those invited travelled to Warsaw and stayed there one night. The next day they were transported by bus to some place found by magician Władek where there was food and accommodation, modest but sufficient. One could meet in this way with famous Russian scientists as Barenblatt, Zel'dovich, Ladyshenskaya and others. The fluid dynamics community is greatly indebted to Władek Fiszdon for organizing these Symposia. Unfortunately, his health condition does not allow him to be here today. From this place I would like to thank him for all he did for Fluid Mechanics in this way.

The first time that I was invited to participate in such an event was in 1969 in Kazimierz (not named after my friend and colleague Kazimierz Sobczyk who will present the Closing Lecture next Friday). George 
Batchelor was a key figure in these Symposia. He had great authority (he was a Foreign Member of the Polish Academy of Sciences), Władek Fiszdon asked his advice whom to invite and he was always very relaxed and willing to lecture on everything that he was working on. I remember very well that he gave a lecture on the sedimentation problem on which he was working at the time and what was to become the subject of his celebrated paper "Sedimentation in a dilute dispersion of spheres" [1]. This concerns the velocity with which a cloud of heavy particles sedimentates in a fluid. The, until that time unsolved, difficulty in this and similar problems is that the velocity which a small particle induces in its vicinity falls off very slowly, as the reciprocal distance from its center. The calculation of the average sedimentation speed results, because of this in not uniformly convergent integrals, with which J.M. Burgers struggled already in the 1930 's. George found a way, a renormalization, to overcome this difficulty. His renormalization technique has found wide application in other areas. His presentation in Kazimierz induced me to think about the analogous problem where a cloud of bubbles rises under buoyancy.

\section{Air and Water}

The flow around a bubble is, to a good approximation, a potential flow. The velocity which one bubble induces in another falls off as (distance from centre to centre) ${ }^{-3}$. In contrast with the falling particle inertia effects are here dominant, the Reynolds number is large. This (distance $)^{-3}$ behaviour is faster than that with the falling particle but not fast enough to overcome difficulties with not uniformly convergent integrals. When a bubble is accelerated, the surrounding liquid exerts a reaction force on the bubble, which is proportional to the acceleration. The multiplying factor has dimension of mass and is called virtual or added mass, because in calculations it can be treated as a virtual mass of the bubble which is itself of course almost massless. It appears that this mass depends on the presence of nearby bubbles in a manner which gives rise to convergence problems. Consider $N$ bubbles in a configuration $C_{N}$ with probability density $P\left(C_{N}\right)$. When there is always one bubble in the point $\boldsymbol{r}_{0}$, such a configuration is indicated with $C_{N-1} \mid \boldsymbol{r}_{0}$ and the corresponding probability density with $P\left(C_{N} \mid \boldsymbol{r}_{0}\right)$.In the course of the calculation one needs to know the average velocity in the centre of a bubble in the presence of all the others, and with respect to the volume velocity $\boldsymbol{U}_{0}$ of the suspension,

$$
\langle\mathbf{u}\rangle-\mathbf{U}_{0}=1 / N ! \int\left\{\mathbf{u}\left(\mathbf{r}_{0}, C_{N}\right)-\mathbf{U}_{0}\right\} P\left(C_{N} \mid \mathbf{r}_{0}\right) d C_{N} .
$$


For low concentration by volume one considers, just as in the case of dilute gases, the interaction between two particles only or, in this case, bubbles. Then Eq. (1) becomes

$$
\langle\mathbf{u}\rangle-\mathbf{U}_{0}=\int\left\{\mathbf{u}\left(\mathbf{r}_{0}+\mathbf{r}, \mathbf{r}_{0}\right)-\mathbf{U}_{0}\right\} P\left(\mathbf{r}_{0}+\mathbf{r} \mid \mathbf{r}_{0}\right) d^{3} \mathbf{r} .
$$

The quantity in curly brackets in Eq. (2) behaves at large distance $r$ as $r^{-3}$ and therefore the integral does not converge. The essence of Batchelor's renormalisation technique starts in this case with noting that if in Eq. (1) we take just $P\left(C_{N}\right)$, that means when we consider the average velocity in a point whether in fluid or in gas, then the result is zero,

$$
0=1 / N ! \int\left\{\mathbf{u}\left(\mathbf{r}_{0}, C_{N}\right)-\mathbf{U}_{0}\right\} P\left(C_{N}\right) d C_{N} .
$$

When reduced to the interaction of two bubbles also this integral does not converge. The only difference with the right-hand side of Eq. (1) is that there is in the latter always a bubble in $\boldsymbol{r}_{0}$ and in Eq. (3) sometimes. However we know the exact result Eq. (3). Now we subtract Eq. (3) from Eq. (1). Since in the absence of long-range order in the suspension we have at a large distance from $\boldsymbol{r}_{0}$

$$
P\left(C_{N} \mid \mathbf{r}_{0}\right)=P\left(C_{N}\right)
$$

the difference of the two integrals converges when the configuration is reduced to two bubbles and this overcomes the problem because we are left with the calculation of the integral

$$
\int\left\{\mathbf{u}\left(\mathbf{r}_{0}, \mathbf{r}_{0}+\mathbf{r}\right)-\mathbf{U}_{0}\right\}\left\{P\left(\mathbf{r}_{0}+\mathbf{r} \mid \mathbf{r}_{0}\right)-P\left(\mathbf{r}_{0}+\mathbf{r}\right)\right\} d^{3} \mathbf{r},
$$

which is now convergent. Although this problem could be solved, [2], the general problem to understand the dynamics of air-water mixtures is today far from being solved.

Particle - liquid flow can either show random configurations or fixed configurations. With air and water many more topologies are possible. I mentioned as a first example the bubbly suspension, a common device in the chemical industry where it serves as a reactor column.

Some more examples are:

- Niagara Falls (American and Canadian).

Air is first entrained by river water falling down and mixes with this during its fall. The air leaves eventually together with only a little bit of water, forming with it a spray, or mistflow. There are in fact 


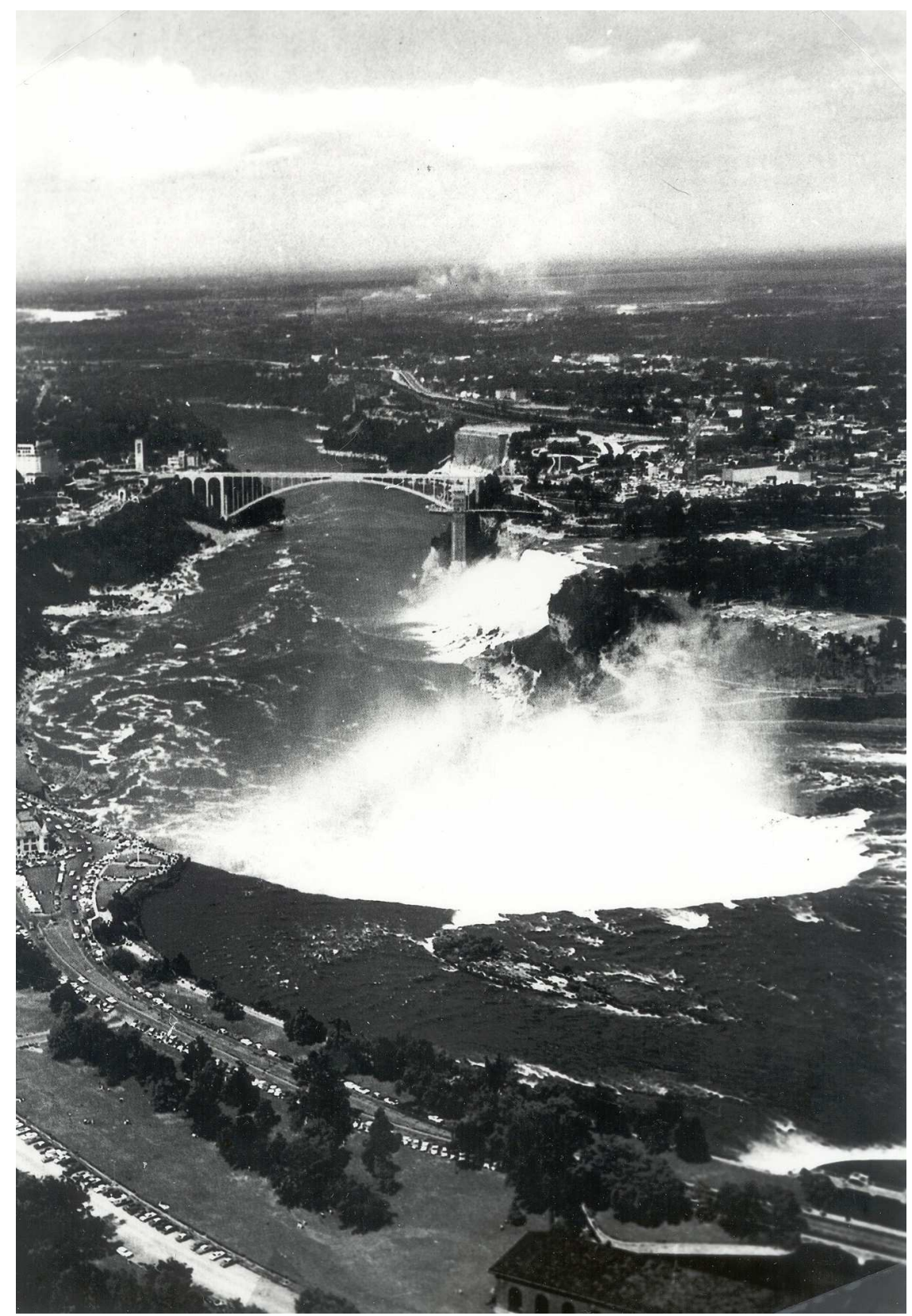

Figure 1. The Niagara Falls, in the foreground the horseshoe-shaped Fall on the Canadian side, and the American Fall in the background. 
two falls, one on the Canadian side, with the shape of a horseshoe, and one on the American side. Although the picture is not very good, you can clearly see that with the Canadian fall water droplets are in the upward flow entrained with the air above the original level from where they originated. They derive some energy from the air. This does not happen at the American fall. The reason seems to me that there is, in the restricted space available for the downflow of the Canadian one, a pressure built up pushing a strong upward air flow entraining droplets.

- Breaking wave with trapped air.

In the case of a breaking wave air is trapped in the overturning wave. This entrained air plays an important role in the dynamics of the wave, and of its impact on walls. A striking effect of the trapped air is a tremendous change in the compressibility of the mixture. Even an air concentration of a few percent dramatically alters the sound velocity which is directly related to the compressibility. This can be made clear as follows.

Denoting the velocity of sound of the mixture with $c_{m}$, we have from thermodynamics

$$
\left(c_{m}\right)^{2}=(d p / d \rho)_{s},
$$

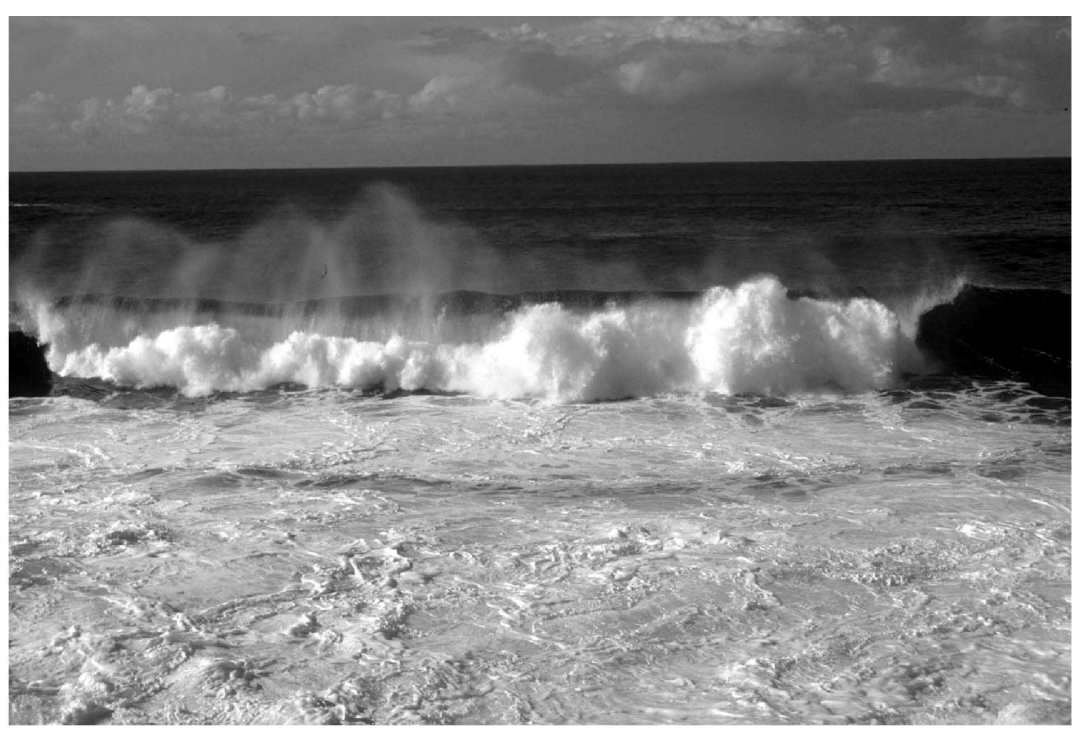

Figure 2. A breaking wave at Coogee Beach, Sydney, Australia. Photograph taken by D.H. Peregrine, University of Bristol, and reproduced here with his permission. 
where $p, \rho$ and $s$ denote pressure, density and entropy respectively. The density is made up from that of air, with volume fraction $\alpha$, and that of water, with volume fraction $1-\alpha$. Referring to air and water with subscripts $a$ and $w$ respectively, we have therefore

$$
\rho=\alpha \rho_{a}+(1-\alpha) \rho_{w} .
$$

We neglect a possible velocity difference between air and water. Then the mass of air in a unit mass of the mixture is constant, whence

$$
\rho_{a} \alpha /\left\{\rho_{a} \alpha+\rho_{w}(1-\alpha)\right\}=\text { constant. }
$$

We assume further that the pressure in water and air is the same (later we shall see when this ceases to be realistic). Then it follows from Eq. (4)-Eq. (6) that for $\alpha$ not too close to zero or to unity

$$
\left(c_{m}\right)^{2}=\gamma p / \rho_{w}(1-\alpha) \alpha,
$$

$\gamma$ being the ratio of specific heats of the air. In Fig. 3 graphs of the complete expression are shown for various ambient pressures. Even with a volume concentration $\alpha$ of air of one percent, the velocity of sound is in an air-water mixture only $100 \mathrm{~m} / \mathrm{s}$, far below the sound speed in either air or water. (Air was, of course, also involved in the generation of the wave. That is an old problem in hydrodynamics.

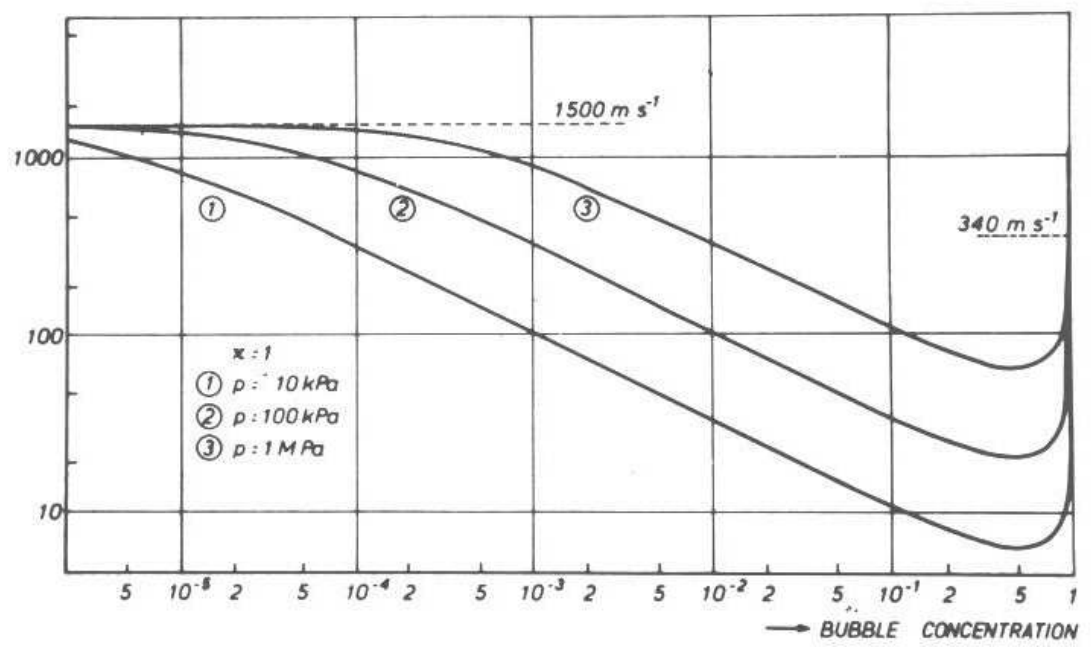

Figure 3. The sound velocity, $c$, in a mixture of air and water. The air concentration by volume $\alpha$ is indicated along the horizontal axis, the sound velocity along the vertical axis. 
In the last 50, or so, years much has been clarified but it is not completely solved.)

The air trapped in water has also a profound effect on the radiation of sound when the flow is turbulent. The late Sir James Lighthill has shown in one of his finest contributions to fluid mechanics, [3], that turbulence produces, inefficiently, quadrupole sound. The presence of air gives a new, by far dominating, monopole contribution, which leads as shown by Crighton and Ffowcs Williams [4], for not too low void fraction, to a sound emission larger by a factor $\left(c_{w} / c_{m}\right)^{4}$ which can be for air and water of the order $10^{6}$ or an intensity increase of $60 \mathrm{~dB}$. Think about this when you hear these waves speak!

- Cavitation.

Another two-phase situation is encountered in cavitation, for example at a hydrofoil, see Fig. 4. Due to the low pressure in the flow along the hydrofoil, a propeller blade, microscopic nuclei become unstable and grow to macroscopic size. In a region of higher pressure these bubbles, filled with air and vapour, collapse again and may cause at the final stage of the collapse considerable damage to the blade.

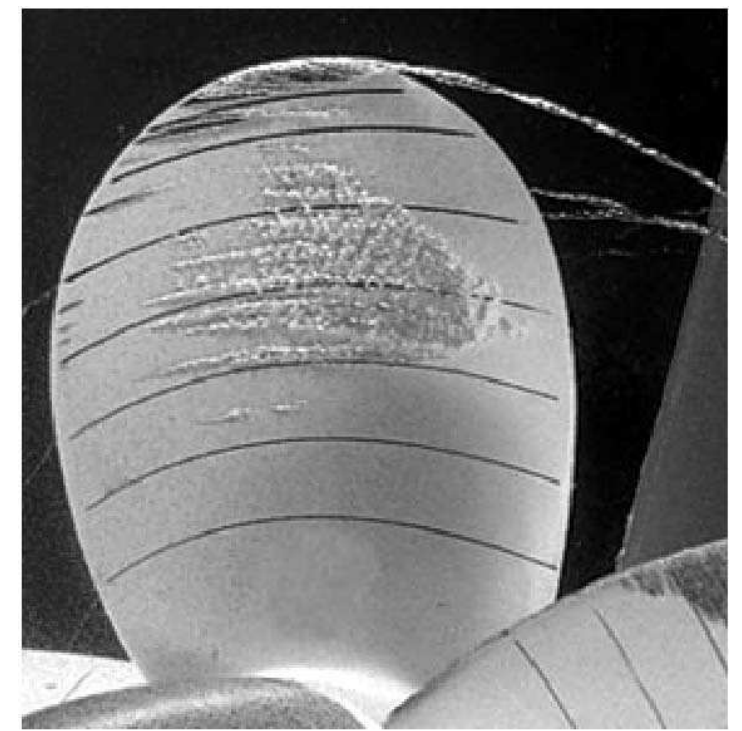

Figure 4. Cavitation on a ship's model propeller turning in a water tunnel. There is cavitation on the blade but also in the tip vortex. Courtesy of the Maritime Research Institute of the Netherlands (MARIN) at Wageningen. 
In all these cases there is interaction between the gaseous phase and the liquid phase and the title of my lecture refers to this. In a further part of the lecture I shall in a symbolic way imagine the air which is above as the domain of fundamental research and the water, down below,as applied science, which will give opportunity to share some thoughts about science and engineering with you.

In the spray above the Niagara waterfall we have a lot of air and some water, in the case of a bubbly suspension we have a lot of water and some air. At both ends there are unsolved problems forming a challenge for fluid mechanics.

\section{Bubbly Flow}

Compared with the Niagara fall, the rising suspension looks quiet and peaceful. But lo and behold what happens when we increase, with bubbles of about $1 \mathrm{~mm}$ radius, the void fraction to about $25 \%$. A violent transition to slug flow occurs. (During the presentation a video of the transition to slug flow was shown). Both phenomena, the homogeneous rise at low concentration and the transition to slug flow are ill understood.

Let us start with the former. The interest in bubbles has always been great. Bubbles smaller than about $0.8 \mathrm{~mm}$ radius rise in a straight line. For example bubbles rising in champagne or beer. The application in champagne is due to Dom Pérignon who was a contemporary of Sir Isaac Newton. Their simultaneous occupations are described by Michel Onfray [5] as "while the first (Pérignon) prepares beverages with rising bubbles, the second (Newton) derives formulas based on falling fruits" (my translation from the French text). This quotation from the chapter "Une petite théorie des bulles" of Onfray's book illustrates on a day like this very appropriately the unity of mechanics.

Bubbles with a radius above $0.8 \mathrm{~mm}$ do not rise linearly in water but perform spiralling or zigzag motion, in contrast to falling particles which fall in a straight line. This was already known to Leonardo da Vinci, who made a sketch of what he saw, Fig. 5, and is therefore called nowadays Leonardo's Paradox, see e.g. Ohl, Tijink and Prosperetti [6].

Recently, see e.g. de Vries et al. [7], it has been observed that these spiralling bubbles have a wake trailing behind them consisting of two vorticity bearing threads, see Fig. 6 . The relevant bifurcation has been also described numerically, Maugin \& Magnaudet [8], but the underlying physical mechanism is not yet understood.

The flow around a bubble rising in clean water is well described by potential flow supplemented with thin boundary layers. These, of the 


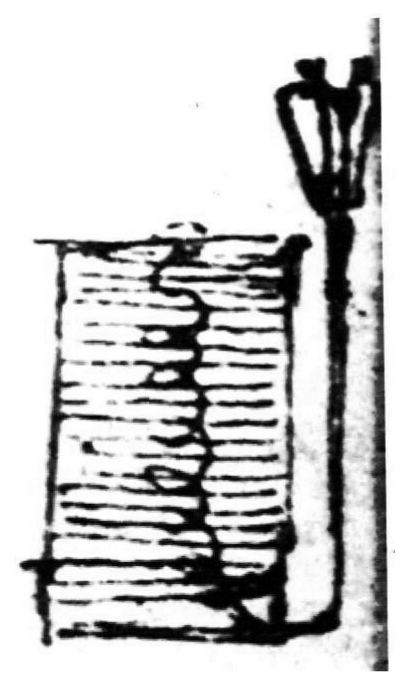

Figure 5. Sketch by Leonardo da Vinci of a spiralling bubble (Courtesy of Musée du Louvre, Paris). The "Paradox", for further details see [6], is in the fact that a falling particle has a straight trajectory but a rising bubble - a spiralling path.

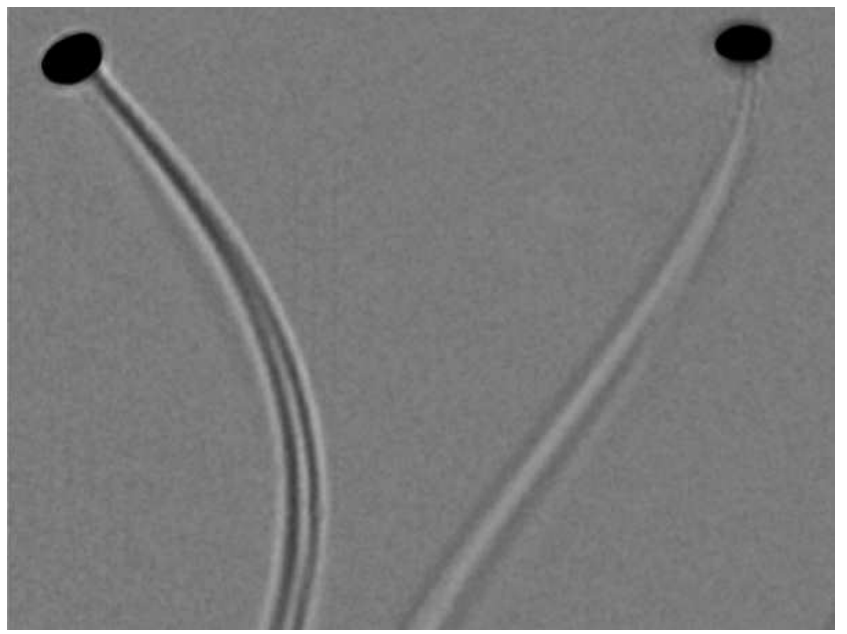

Figure 6. Two mutually perpendicular projections of a bubble spiralling in hyperclean water. The effective bubble radius is about $1 \mathrm{~mm}$. Clearly seen is the double -threaded wake behind the rising bubble. Courtesy of Christian Veldhuis. 
same thickness as with rigid bodies, have now only to overcome the difference in shear stress between the outer flow and the zero stress at the interface. Just as in the study of dilute gases, it is natural to start with looking at binary interactions. Numerical work and analytical work with this model unfortunately predict clustering of bubbles and not the homogeneously rising bubbles as observed in experiments. This can be made plausible as follows: Imagine two bubbles with their line of centres making, at time $t=0$, an angle of more than 36 degrees with the vertical direction which is parallel to gravity. The hydrodynamic forces are such that after a time of order $a / V$, where $a$ is the bubble radius and $V$ the terminal velocity, the line of centres is horizontal. This is therefore a statistically highly probable situation. But in this configuration there is only an attractive force between the bubbles. They bounce for a while but even when this is a purely elastic collision, the motion gets exhausted by viscous friction, which explains the clustering in the numerical simulation.

How in reality the lack of repulsive forces, the basic reason for the clustering, is overcome is another unsolved problem of two-phase flow. I have made recently [9]) the suggestion that the above mentioned twothreaded wakes could play a role here.

To continue with unsolved problems in two-phase flow I recall the transition to slug flow. Also this awaits an explanation. There is an analogeous phenomenon in fluid beds. There the homogeneous bed becomes unstable, big gas bubbles are formed, as a result of the instability of concentration waves. It has been thought for some time that transition to slug flow is similarly due to instability of such waves. Evidence, Lammers \& Biesheuvel [10] shows this not to be the case. The instability of concentration waves (or void fraction waves) occurs but before slugs appear, there is an intermediate flow regime characterized by a pointed transverse velocity and concentration distribution.

You might have the impression by now that in two-phase flow there are mainly riddles and unsolved problems. This is certainly not the case. I will illustrate this with two examples. The first is about expansion waves in a two-phase flow. We have seen that the bubbly suspension has a low velocity of sound. So, we can play at low velocities the whole organ of compressible gases. For example the theory and experiments of waves of finite amplitude. There is, however, an important difference. If pressure changes become very rapid, the relation Eq. (7) for the speed of sound is no longer valid. My compatriot Marcel Minnaert measured in the 1930's the frequency of volume oscillations of small air bubbles in water in an ingenious way, described in [11]. He determined the frequency of the audible popping sound of the bubbles formed in his 
apparatus by means of tuning forks. He also derived a formula for this frequency, $f$, say,

$$
f=1 /(2 \pi a)\left\{3 \gamma\left(p-p_{v}\right) / \rho_{l}\right\}^{1 / 2}
$$

This is for a bubble with radius of $1 \mathrm{~mm}$ about $3 \mathrm{kHz}$. When now in a bubbly suspension pressure changes are not far from this Minnaert frequency, the bubbles do no longer passively follow the pressure changes but pressure differences between the two phases develop due to the inertia of the liquid. As a result the medium becomes dispersive which expresses itself in various ways. One of these is that the velocity of propagation of a wave of finite amplitude depends not only on the amplitude, as is the case for "normal" compressible fluids, but also of the frequency, or wavelength. With weak nonlinearity and weak dispersion the famous Korteweg-de Vries equation is valid for the pressure in the wave. Some time ago we did [12] the following experiment:

At the entrance of a semi-infinite bubbly flow, a time-dependent pressure was established in the form of a rectangular triangle, a shock wave followed by a rarefaction. For this special initial profile the KdV equation can be solved exactly with help of the so-called inverse scattering theory. The evolution in the mixture of this initial profile is into a train of solitons according to this theory. The nice thing about this is that the associated mathematical equation, a Schrödinger equation, has for this particular profile an exact solution in the form of an Airy function and that the number of evolving solitons is equal to the zeros of this Airy function in a certain interval. In the experiment that we did, the shape of the evolving waves was not quite that of solitons (they were still evolving) but the number agreed exactly with the predictions, see Fig. 7.

Another example is directly connected to Minnaert's early finding. Much later it was discovered that also the sound of rain on a water surface is due to bubbles but in a special way.

The falling drop forms a crater in the water which is filled with air. As the crater closes again the air escapes but sometimes a small air bubble is trapped. This produces noise while oscillating in its Minnaert frequency. Experiments, see Oğuz \& Prosperetti [13], with drops of various speeds and sizes show that a bubble is trapped only in a narrow portion of the speed/size plane (see Fig. 8). In nature the speed of the raindrop depends on its size and hence the intersection of this line with the above mentioned area gives the size of the raindrops producing bubbles and accompanying sound. This explains the rather narrow frequency spectrum of rain noise. 


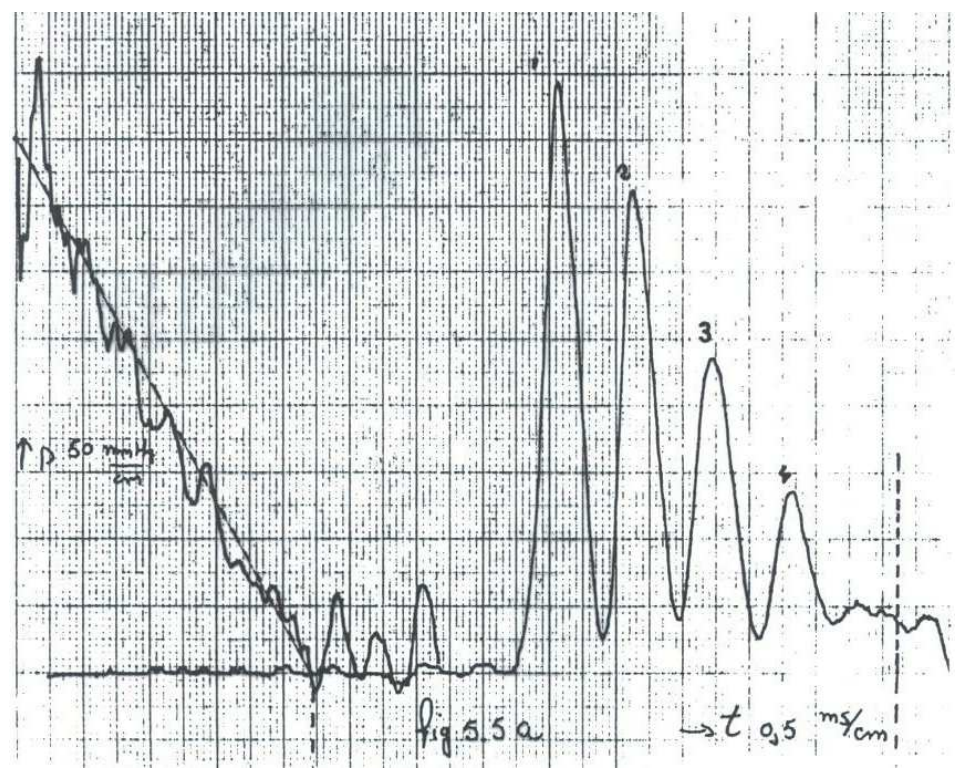

Figure 7. From an initial pressure profile shaped in terms of time as a rectangular triangle by a shock wave followed by an expansion wave, (left-hand side of picture) develops, after the wave has travelled a long distance in the bubbly flow, a series of solitons (right-hand side of picture).

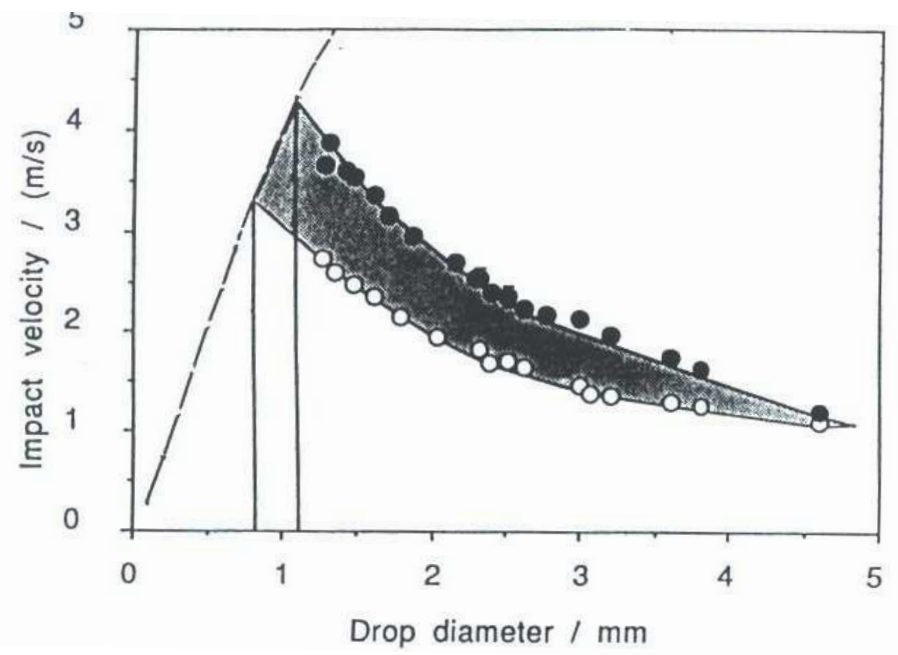

Figure 8. The shaded area bounds that portion out of the diameter/impact plane of falling drops, that marks the occurrence of a trapped air bubble. The broken line represents the relation between diameter and impact velocity of raindrops. Hence the intersection of this line with the shaded area gives the range of raindrops which produce air bubbles and thereby sound. The picture is from [13] and reproduced here with permission of Ann. Rev. Fluid Mech. 


\section{Fundamental and Applied, The Scientist and the Engineer}

I will now use the freedom of the lecturer on an occasion as this one to compare in a symbolic sense the interplay just described with the interplay between fundamental and applied science. Fundamental science high up in the air, the applications down below where the water is. Sometimes the interplay is not obvious. Famous are the words spoken by the German mathematician David Hilbert when he was asked to comment on the relation between pure and applied science. He said "Meine Herren, sie haben gar nichts mit einander zu tun" (they have nothing to do with each other). This points at a lack of appreciation. In my case I have been lucky to see a lot of both in my professional life. Both have their peculiarities of which I shall give two examples. First the " $"$ " and the "=". What do I mean by this? Suppose you are designing a device which includes flow of water, of kinematic viscosity $\nu=10^{-6} \mathrm{~m}^{2} / \mathrm{s}$, with a velocity $U=0.10 \mathrm{~m} / \mathrm{s}$ in a pipe with diameter $D=1 \mathrm{~cm}$. You want to know for the operation of your device, what entry length $l$ is needed for the flow to become fully developed. You turn for advice to a theoretical physicist. He takes his copy of Landau \& Lifshitz, Fluid Mechanics [14], from the shelf in his office and finds on page 150

$$
l / D \sim \text { Re. }
$$

This means that the dimensionless entrance length $l$ is a multiple of order unity times the Reynolds number $U D / \nu$. Landau \& Lifshitz point out that the thickness $\delta$ of the laminar boundary layer along the pipe wall grows, with $x$ running along the pipe axis, as

$$
\delta \sim(\nu x / U)^{1 / 2},
$$

where again $\sim$ means that the boundary layer thickness is a multiple of order unity times the shown quantity. Putting now the thickness equal to the diameter gives Eq. (9) for the entrance length $l$. In this particular case $R_{e}$ is 1000 . You feel uneasy over it and you ask the physicist, do I really need thousand diameters, which is $10 \mathrm{~m}$ ? The physicist does not listen anymore. Your problem is now an engineering problem and he does not care. So, you turn to an engineer, for example R.S. Brodkey, who tells you in his book Brodkey 1967 [15] on page 129 that exact calculation gives

$$
l / D=0.06 \text { Re } .
$$

This is, to your great relief, only 60 diameters or $60 \mathrm{~cm}$.

As another example I mention granular materials. This is nowadays a popular subject in physics. It has been,however, widely studied in 
civil engineering and in mechanical engineering since long ago. In the second part of the last century A.W. Jenike (1914-2003) has dominated research in the field of bulk solids. The research in this field has had results. During the last ICTAM of the last century, Chicago 2000, in one invited lecture, Roux \& Radjaï [16], we read " The quasi-static behaviour of granular materials is already a mature field in which a number of elastico-plastic models reproduce very accurately the available experimental tests. They allow us to design civil engineering structures with confidence". The authors of these lines come from civil engineering. At the same ICTAM there was another invited lecturer, Goldhirsch [17], a physicist, from whose lecture I quote: "Granular matter is often described as "unpredictable", "irreproducible" or "erratic". These and other adjectives used to characterize granular matter are a clear sign that much is still lacking in our understanding of these "materials" (my italics). In my opinion the physicists could have in this case more appreciation for the work done and results obtained by engineers.

\section{Epilogue}

The great experience in ICTAM is that both fundamental and applied scientists can listen to each other and talk to each other during and outside the many sessions. And in spite of differences of approach, illustrated in the previous Section with some examples, there are many aspects in their work that they share. Whereas consultant firms apply high per-hour rates for every service that they deliver, we all are referees and editors for journals, sit in committees, do work for funding organisations, you name it, without payment or at most a modest compensation for subsistence costs. Why do we do that? There are immaterial rewards in the form of prizes and other signs of recognition. But above all it is out of a sense of duty to the scientific community. The British writer and philosopher Iris Murdoch [18] points out that our sense of duty stems from the fact that we are not perfect beings, "A totally good being would not experience the call of duty, might be said to lack or not need the concept since all acts and decisions would emerge from virtuous insight and its orderly process". But just this sense of duty saves us, according to an Editorial in Science [19], from becoming victims of human frailty. I quote from this article entitled "The Roots of Scientific Integrity": "The system of rewards and punishments tends to make honest, vigorous, conscientious hardworking scholars out of people who have human tendencies of slothfulness and no more rectitude than the law requires". 
With this quotation Mr Chairman, intended to make us all feel good, I come to the end of my presentation. I wish you all an enjoyable and rewarding ICTAM 2004 and I thank you for your attention.

I thank my colleagues of the Physics of Fluids Group of the University of Twente for their helpful comments, Michel Versluis for teaching me Power Point and Raymond Bergmann, Peter Eshuis, and Christian Veldhuis for their expert help in preparing the Power Point version of this lecture.

\section{References}

[1] G.K. Batchelor, Sedimentation in a dilute dispersion of spheres, J.Fluid Mech., Vol.52, pp.245-268, 1972.

[2] L. van Wijngaarden, Hydrodynamic interaction between gas bubbles and liquid, J.Fluid Mech., Vol.77, pp.27-44, 1976.

[3] M.J. Lighthill, On sound generated aerodynamically 1. General theory, Proc.R.Soc.London, Vol.A211, pp.564-587, 1952.

[4] D.G. Crighton and J.E. Ffowcs Williams, Sound generation by turbulent twophase flow, J. Fluid. Mech., Vol.36, pp.585-603, 1969.

[5] M. Onfray, Gourmande La Raison, Grasset et Fasquelle, 1995.

[6] C.D. Ohl, A. Tijink, A. Prosperetti, The added mass of an expanding bubble, J.Fluid Mech., Vol.482, pp.271-291, 2003.

[7] A.W.G. de Vries, A. Biesheuvel, L. van Wijngaarden, Notes on the path and wake of a gas bubble rising in pure water, Intn'l J.Multiphase Flow, Vol.28, pp.1823-1834, 2002.

[8] G. Mougin, J. Magnaudet, Path instability of a rising bubble, Phys.Rev. Lett., Vol.88, 014502- 1-014502-3, 2002.

[9] L.van Wijngaarden, Bubble velocities induced by trailing vortices behind neighbours, Submitted to J.Fluid Mech., 2004.

[10] J.H Lammers, A. Biesheuvel,, Concentration waves and the instability of bubbly flows, J.Fluid Mech., Vol.328, pp.67-93, 1996.

[11] M. Minnaert, On musical air bubbles and the sound of running water, Phil. Mag., Vol.16, pp.235-245, 1933.

[12] L.van Wijngaarden, Evolving Solitons in Bubbly Flows, Acta Applicanda Mathematicae, Vol.39, pp.507-516, 1995.

[13] A. Prosperetti, H.N. Oğuz, The impact of drops on liquid surfaces and the underwater noise of rain, Annu. Rev. Fluid Mech. Vol.25, pp.486-537, 1993.

[14] L.D. Landau, E.M. Lifshitz, Fluid Mechanics, Pergamon, 1959.

[15] R.S. Brodkey, The Phenomena of Fluid Motion, Addison-Wesley, 1967.

[16] S. Roux, R. Fahrang, Statistical Approach to the mechanical behaviour of granular media, In Mechanics for a New Millenium [Eds.] H. Aref, and J.W. Phillpps, pp.181-197, Kluwer Academic Publishers, 2001. 
[17] I. Goldhirsch, Kinetic and continuum descriptions of granular flows, Mechanics for a New Millenium, Eds. H. Aref and J.W. Phillips, pp.345-359, Kluwer Academic Publishers, 2001.

[18] I. Murdoch, Metaphysics as a guide to morals, Penguin Books, 1993.

[19] Editorial Essay "The roots of scientific integrity", Science, Vol.139, pp.3161, 1963. 